\title{
A Comprehensive Review of Numerical Weather Prediction Models
}

\author{
Rashi Aggarwal \\ Jamia Millia Islamia University, India
}

\author{
Rajendra Kumar,Ph.D \\ Assistant Professor, Department of Computer \\ Science \\ Jamia Millia Islamia University, India
}

\begin{abstract}
Weather forecasting has been an area of considerable interest among researchers since long. In particular, precipitation has been found to be interesting because of its chaotic nature and also because of the direct impact it has on the society. Even after the invention of complex Coupled Numerical Weather Prediction Models, the errors in prediction have been found to be of significant magnitude. The present study aims at investigating all the aspects of error dynamics in dynamic and statistical predictions, and reviews these two prediction models on the basis of errors arising due to initial conditions and understanding of physical processes generating with time series.
\end{abstract}

\section{Keywords}

Artificial Neural Networks, weather forecasting, time series analysis.

\section{INTRODUCTION}

Weather has attracted the mankind since time immemorial. The unpredictable nature of the weather and its socioeconomical importance makes it interesting for the researchers to device accurate weather forecasting algorithms. There are widely two popular approaches to weather forecasting numerical (dynamical) and statistical (including the Artificial Intelligence methods). Numerical weather prediction refers to dynamic modelling of the atmospheric system. This type of modelling requires huge computational resources as well as full understanding of the subject. The predictability of such systems are further complicated by the uncertainty in the initial conditions. Ensembling forecast has been in practice since long to account for the errors due to initial conditions. Multi Model Ensembling (MME) has been recently adopted by researchers to take into account the errors due to lesser understanding of the subject.

Statistical prediction models such as the Artificial Neural Networks (ANN) tend to model the dynamical behaviour of the system based on past information. The errors in such techniques can be due to wrong initializations as well due to wrong topology.

\subsection{Issue of uncertainty in weather prediction}

The possibility of accurate weather prediction is still a distant dream for researchers. The sensitive dependence of the weather system on initial conditions does not render the

system to be modeled by deterministic methods. Such systems are called chaotic systems. It may, nevertheless, be useful to have a system which may be able to predict if catastrophic events will occur even if the scientific understanding of the subject remains limited. Therefore the creation of a model with dynamical characteristics similar to the unknown model generating the time series produced by these systems could help in the prediction of such systems. Artificial Neural Network is one such system.

\subsection{Application of Artificial Neural Networks (ANN) in weather forecasting}

Artificial Back-propagation Neural Networks (ANNs) are statistical models which have been widely used for time series prediction. During the last decade there have been successful applications of these models in the meteorological domain. Hsieh and Tang (1998) ${ }^{[22]}$ have summarized the methodology of application of ANN models to meteorology and oceanography. They have applied $\mathrm{NN}$ models to oceanography and meteorology, especially in large scale low frequency studies and examined three main obstacles i.e., a) Non-Linear instability with short data records, b) large spatial data fields, c) difficulties in interpreting the non-linear NN results. Obstacle 1 was removed by ensemble averaging and methods like penalty, pruning and non-convergency. It was observed that cost function used to get stuck in one of the local minima and hence optimal search was not guaranteed. Also, if number of neurons increased or when data record used to get shortened then search usually ended up at one of the local minima. Obstacle 2 was overcome by using PCA method through which the dimension of large spatial data fields reduced to a great extent and only main features were extracted. For obstacle 3, the mysterious hidden layer could be given a phase space interpretation, and spectral analysis aided in understanding the nonlinear NN relations. Gyanesh and others $(2012)^{[7]}$ have presented a comprehensive review of the application of ANN model to meteorology and oceanography. 
Sahai et. Al (2000) ${ }^{[1]}$ used two approaches for the prediction of ISMR using parameters such as the regional conditions, EI Nino-southern oscillation indicators, crossequatorial flow and global conditions through the analysis of linear correlation with ISMR. The first approach was that of linear regression and the second one was that of the ANN model. It was observed that the predictions by ANN models have much longer lead time as compared to conventional approaches such as the linear regression.

One day ahead forecasts of the hourly ozone concentration for the rural and urban site have been carried out using the ANN model (Ballester et. Al., 2002) ${ }^{[4]}$. The model utilized past and previously predicted information of inputs exclusively, thus it has been treated as a genuine 24h advance $\mathrm{O} 3$ predictive model with neural networks. It was concluded that prediction performance of the models depend significantly on the cluster number.

Predictability of sea surface temperature anomalies in the Indian ocean was analyzed by Tripathi and others (2008) [10]. Reynold's reconstructed SST data set was used for the study. The data was divided into twelve sets - one data set for each month. A comparison with linear regression model was made and it was observed that both the models had similar predictability skills when broad statistical analysis was done. However, it was observed that during certain months the ANN gave significantly good predictions when the regression model could not make any prediction at all.

Tripathi and others (2008) ${ }^{[10]}$ have used the Southern Indian ocean SST indices for early prediction of Indian summer monsoon rainfall using the ANN model. These indices were for the (i) Central Southern Indian Ocean (CSIO), (ii) Northwest of Australia (NWA), (iii) Southern Indian Ocean (SIO) and (iv) Antarctic Circumpolar Current (ACC). In the present chapter, ANN is used to study the predictability of the Indian summer monsoon with four indices individually as well as in various combinations. It was found that two combinations of SST indices, (SIOI + ACCI) and (CSIOI + $\mathrm{NWAI}+\mathrm{SIOI}+\mathrm{ACCI})$, show best predictive skill when used collectively. They that the performance of the ANN model is better than the corresponding regression model in the prediction of ISMR indicating that the relationship between ISMR and SST indices are non-linear in nature.

Radhika and Shashi (2009) ${ }^{[23]}$ have applied Support Vector Machines (SVM's) for weather prediction. Mean square error was taken as a performance measure. It was observed that irrespective of the order, SVM performed better than MLP as root mean square error in SVM was much smaller. It was concluded that parameter selection in all cases of SVM has a significant effect on the performance of model. Recently ANN technique has been used for rainfall forecasting in Alexandria, Egypt (Shafie et.al., 2011) ${ }^{[5]}$. Comparison with linear regression models was made on the basis of mean error (BIAS), mean absolute error (MAE), root mean square error (RMSE), and the correlation coefficient (CC) and it was observed that both the models had the ability to predict the extreme values, maximum and minimum values. However, the ability to predict the mid-range values is better for the ANN model.

Shukla and others $(2011)^{[15]}$ have used the ANN model for the prediction of ISMR using various Nino indices as predictors. Correlation analysis is done to see the effect of SST indices of Niño-1+2, Niño-3, Niño-3.4 and Niño-4 regions on ISMRI with a lag period of 1-8 seasons. Significant positive correlations, with confidence level above 99\%, are found between ISMRI and (i) Niño-3 index with a lag of 4 (June-July-August) and 5 (March-April-May) seasons, (ii) Niño-3.4 index with a lag of 4 and 5 seasons and (iii) Niño-4 index, with a lag of 5 seasons before the onset of monsoon. These SST indices are used for prediction of ISMRI using multiple linear regression and Artificial Neural Networks (ANNs) models. A comparative examination of the results suggests that the ANN model has better predictive skills than all the linear regression models investigated, implying that the relationship between the Niño indices and the ISMRI is essentially non-linear in nature.

Aggarwal and others (2012) ${ }^{[2]}$ have used the ANN model to predict maximum and minimum temperatures. Mechgoug and others (2012) ${ }^{[14]}$ have used the ANN model for the prediction of monthly ozone and carbon dioxide concentration time series. They have used the genetic algorithm for automating the design of neural networks.

\subsection{Numerical Weather Prediction and Multi-Model Ensembling (MME)}

The numerical climate models can be broadly classified (Mason, 2008) ${ }^{[11]}$ as the two-tiered climate models and the single-tier or fully-coupled climate models. In two tiered forecasting to model the climate, only the atmosphere is modelled dynamically while the values of various parameters in the lower boundary are specified manually. If these models are used for the purpose of forecasting the atmospheric variables, future values of the boundary conditions have to be specified and hence forecast prior to the forecasting of the atmospheric variables. Thus there is a two-tiered forecasting: the forecast of the boundary conditions and then, using those forecast, the forecast of the atmospheric variables (Bengtsson, $1993)^{[3]}$. In fully-coupled models, all the relevant components are modelled and they generate the forecast of the atmospheric variables and the boundary conditions simultaneously. There are computational advantages of using the two tiered coupled model than the state-of-the art one tier coupled model (Mason, $2008)^{[11]}$. This creates a need for statistical modelling.

As has been pointed out earlier, the predictability of dynamical systems are largely governed by two factors - (i) the uncertainty arising out of initial conditions and (ii) poor understanding of the actual process in question. Ensemble forecasting has been in practice since long to account for the errors due to initial conditions. Multi Model Ensembling (MME) has been recently adopted by researchers to take into account the errors due to lesser understanding of the subject.

Super ensemble architecture for numerical weather prediction was proposed by Krishnamurti and others (2000) [19]. This idea was an extension of the traditional ensemble mean forecasting which could remove errors associated with initial conditions (Palmer et al., 2004 ${ }^{[12]}$, Suzuki et. al., 2004 ${ }^{[18]}$ ). The idea was to first take the ensemble forecast of individual models by using the perturbation theory and then take an ensemble of the ensemble forecasts. Thus the superensemble model was capable of taking into account the errors caused by wrong initializations as well as poor understanding of the concerned deterministic system. This was called the Multi Model Ensembling (MME). In seasonal climate forecasts, the ensemble forecasts are normally 
constructed by using initial perturbations from adjacent start dates.

Krishnamurthy and others (2000) ${ }^{[19]}$ have demonstrated the performance of multimodel ensemble forecast analysis that showed superior forecast skills as compared to other individual models used. The parameters used for comparison among these models include global weather, hurricane track and intensity forecasts, and seasonal climate simulations. To access how many models are minimally needed to improve the skill of multimodel superensemble, one to seven models were examined sequentially. It was observed that ensemble mean error increased as the number of ensemble members increased beyond three. The approach to superensembling assigns different weights to each model depending upon its ability to predict accurately.

The concept of superensembling was further investigated by Yun and others (2004) ${ }^{[21]}$ wherein MultiModel Synthetic superensemble Algorithm was used for seasonal climate prediction using DEMETER Forecasts (Developed by European Center for Medium Range Weather Forecasts, ECMRWF). The objective was to design a multimodel ensemble for seasonal climate prediction using oceanatmosphere coupled models. The approach proposed here is the weighted multi-model superensemble which is a post processing product of multi-model forecasts. The multimodel data set used in this study is the output of seven global coupled ocean-atmospheric models from DEMETER. Each model in itself run in ensemble model based on 9 initial conditions from each start date. Rai and others $(2008)^{[13]}$ have used the MME technique for the investigation of potential of combining the multiple neural network models for time series forecasting and further studied the predictive skills of each model and the multi model ensemble of DEMETER system for the zonal and meridional winds for the region between Madagascar and western Australia covering some parts of sub tropical Indian ocean dipole. It was observed that prediction of different models varied regionally. Root mean square error was calculated for individual models as well as multi model ensemble and it was further demonstrated that multi model ensemble reduced root mean square error to a great extent but still it could be treated as the best model since many individual models also showed lesser root mean square error. Velazquez and others (2011) ${ }^{[9]}$ have investigated whether or not a multi-model approach improves hydrological ensemble forecasting. The study is based on a meteorological ensemble prediction system (M-EPS) coupled with a hydrological model searches to capture the uncertainties associated with the meteorological prediction to better predict river flows. It aimed at evaluating and comparing the performance and the reliability of different types of hydrological ensemble prediction systems (H-EPS), when ensemble weather forecasts are combined with a multi-model approach. Results obtained for each catchment confirmed the superiority of the H-EPS for one area whereas best results were achieved by deterministic and probabilistic simulations.

Bhowmik and Durai (2012) ${ }^{[17]}$ have developed a multimodel ensemble based district level medium range rainfall forecast system for the Indian region making use of model outputs of state-of-the art global models from the five leading global NWP models. To evaluate the performance of district level forecasts, skill score-Probability of Detection is considered. The results demonstrated that performance skill of
Day 1 forecast of district level forecast for rainfall was considerably good for all the considered states. It was concluded that all these models have the capability to capture large scale rainfall features of summer monsoon, such as heavy rainfall belt along the west coast. It is clearly demonstrated that MME is considered superior than other models.

\subsection{Ensembling in statistical scenario}

In dynamical modelling the errors in initial conditions arise out of incorrect observations, incorrect calibration or because of finite memory of the digital system available with the researcher. These are handled by applying perturbations to the initial observations. Several initializations are necessary for chaotic systems because errors tend to grow with time. On the other hand while modelling a phenomenon with statistical technique, modelling the input-output relationship between the predictor and the predictand is taken into account. The inputs are presented to the system in a discrete system and the output at previous time step does not make any impact on the output of present state. There are two aspects of this situation -

1. The present output of the statistical model in consideration does not take into account the previous output.

2. The present output of the statistical model in consideration takes into account the previous output.

In the first case it may appear that initial condition errors may not play any role in analyzing the performance of the model as it does not takes into consideration the dynamical aspects of the system. Example of such systems are the regression models and the Backpropagation networks without momentum parameter. However, this may not be so. This is because the outputs of a statistical model depends on the input-output relationship of the predictor and the predictand which in turn is determined by the actual dynamical laws. Thus it is not a priori obvious whether or not the initial conditions errors play any role in statistical predictions. Further, when the relationship is modelled by the learning mechanism as in the artificial intelligent systems, the concept of local minima comes into play. This may also be attributed to the uncertainties in the initial conditions.

In the second case mentioned above, the uncertainties in the initial conditions may actually amplify the errors with time as in the case of dynamical systems. Examples of such systems are the Recurrent Neural Networks and the Backpropagation network with momentum parameter introduced.

Thus it becomes pertinent to investigate all these aspects of error dynamics in statistical predictions as a comprehensive study. The present study aims at this. Multi model ensembling in this scenario refers to the incorrect architecture which is in correspondence with the limited understanding of the problem in dynamical systems.

Ensembling of ANN models with limited scope of investigation has been undertaken by researchers of this field. Maqsood and others (2004) ${ }^{[8]}$ have studied the applicability of ensemble of ANN for weather prediction in southern Saskatchewan, Canada. It was observed that generalization ability of neural network systems can be significantly improved through an ensemble of neural network by training many NN's and then combining the component predictions. 
Sahai and others (2008) ${ }^{[16]}$ have transformed previously used empirical model to construct a large ensemble of models to deliver useful probabilistic forecast of AIR. The forecast system was found to be capable of predicting monsoon droughts with higher confidence.

Gheyas and others (2012) ${ }^{[6]}$ have proposed a novel homogeneous neural network ensemble approach called Generalized Regression Neural network ensemble for forecasting univariate time series which is a hybrid of various machine learning algorithms. It was found that GEFTS proved better at both short and long horizons as compared to other conventional algorithms. Also it performed better in the conditions where time series were not seasonalized. Ensemble architecture of GEFTS reduced the curse of dimensionality by maintaining an adequate ratio of test cases per variable.

Krasnopolsky and Lin (2012) ${ }^{[20]}$ introduced a non linear NN ensemble approach to improve $24 \mathrm{H}$ multi model ensemble precipitation forecast. This approach demonstrated non linear correlation between ensemble members and optimal forecast represented by a non linear NN ensemble mean. Various techniques has been employed to predict weather. With dynamic models, accuracy was achieved but involved high computational effort. When $\mathrm{NN}$ ensemble was compared with simple linear ensemble, it was observed that $\mathrm{NN}$ ensemble reduced high bias at low precipitation level, low bias at high precipitation level. Also, it sharpened features giving a more accurate result.

Apart from meteorology and oceanography, ANN ensembling has been used in forecasting the exchange rate (Zhang and Berardi, 2001) ${ }^{[24]}$. The data considered was weekly exchange rate data between the British pound and US dollar from 1976 to 1994. a single hidden layer network was chosen as it is capable of modelling any type of functional relationship. Then, neural networks were combined and got trained with initial random weights and kept the best network to reduce the local minima effect. The data was divided into the training, validation and test sets in random as well as serial fashion. These models were then combined to create a single ensemble forecast. The results showed that by appropriately combining different neural networks, forecasting accuracy of individual networks can be largely improved.

\section{SUMMARY AND CONCLUSION}

This study concentrates in the capabilities of dynamic and statistical models for time series analysis. It has been concluded that errors in initial conditions can be dealt effectively by slightly perturbing the observations using sound perturbation theory techniques. The model outputs with slightly varying initial conditions can be averaged to get the final output. It has been observed that doing so improves the forecast skills. However, the averaging may be done by taking into account various factors. The errors arising out of limitations in the understanding of the phenomenon and hence in the formulation of the model have been tried to be handled by doing the Multi Model Ensembling.

Although the dynamic modelling is a must for better understanding of the actual processes, it is not easy to implement owing to the huge amount of computing resources involved. This makes the process expensive. Statistical modelling, on the other hand, requires less computing resources and, hence, is less expensive. Thus it becomes pertinent to investigate all these aspects of error dynamics in statistical predictions as a comprehensive study.

The actual effect of neural network ensemble forecast on the basis of initial conditions of the ANN and also on the basis of topological organization could be further explored as in the case of dynamical modelling. The effect of super-ensembling of neural network on sensitivity to initial conditions and network topology could be addressed. The performance shall be analyzed with simulated annealing algorithm for reaching global minima.

\section{REFERENCES}

[1] A.K Sahai, M.K Soman, V.Satyan (2000). All India summer monsoon rainfall prediction using an artificial neural network. Springer-Verlag, Climate Dynamics vol.16 pg. 291-302

[2] Abhishek Aggarwal, Vikas Kumar, Ashish Pandey and Imran Khan (2012), An application of time series analysis for weather forecasting, International Journal of Enginnering Research and Applications, ISSN:22489622, Vol. 2, issue 2 974-980

[3] Bengtsson, L., Schlese U., Roeckner, E., Latif, M., Narnett, T.P. and Graham, N.E. (1993), A two-tiered approach to long-range climate forecasting, Science, 261 1028-1029.

[4] E Balaguer Ballester, G Camps i Valls, J.L CarrascoRodriguez, E Soria Olivas, S del Valle-Tascon (2002), Effective 1-day ahead prediction of hourly surface ozone concentrates in eastern Spain using linear models and neural networks, Elsevier, Ecological Modelling 1562741.

[5] El-Shafie, A. H. , El-Shafie, A., El Mazoghi, H. G., Shehata, A. and Taha, M. R. (2011), Artificial neural network technique for rainfall forecasting applied to Alexandria, Egypt, International Journal of the Physical Sciences 6, 1306-1316

[6] Gheyas, I. A. and Smith, L. S.(2012), A novel neural network ensemble architecture for time series forecasting, doi:10.1016/j.neucom.2011.08.005 Neurocomputing,

[7] Gyanesh Shrivastava, Sanjeev Karmakar, Manoj Kumar Kowar, Pulak Guhathakurta (2012), Application of Artificial neural networks in Weather Forecasting: A Comprehensive Literature Review, International Journal of Computer Applications (0975-8887), Vol 51- no.18

[8] Imran Maqsood, Muhammad Riaz Khan , Ajith Abaraham (2004). An ensemble of neural networks for weather forecasting, Springer-Verlag, Neural Comput \& Applic vol. 13 pg.112-122.

[9] J.A Velazquez, F. Anctil, M.H Ramos, C.Perrin (2011), Can a multi-model approach improve hydrological ensemble forecasting? A study on 29 French catchments using 16 hydrological model structures, Advances in Geosciences, doi:10.5194/adgeo-29-33-2011.

[10] K.C Tripathi, Shailendra Rai, A.C Pandey, I.M.L Das((2008). Southern Indian Ocean SST indices as early 
predictors of Indian Summer Monsoon. International Journal of Marine Science, Vol. 37(1),pp. 70-76.

[11] Mason, S.J (2008), From Dynamical Model Predictions to Seasonal Climate Forecasts, (chapter in Seasonal Climate: Forecasting and Managing Risk, edited by inter-annual prediction (DEMETER), Bulletin of American Meteorological Society, 85 853-872.

[13] Rai, S., A. C. Pandey, K. C. Tripathi and S. Dwivedi (2008), Predictive Skill of DEMETER models for wind prediction near Madagascar, Indian Journal of Marine Sciences, 37, 62-69.

[14] Raihane Mechgoug, A. Taleb Ahmed, Lakhmissi Cherroun (2012), Optimization of neural predictor for air pollution, Preceedinds of the World Congress on Engineering vol II

[15] R. P. Shukla, K. C. Tripathi, A. C. Pandey and I. M. L. Das(2011), Prediction of Indian summer monsoon rainfall using Niño Indices: a neural network approach, Atmospheric Research, DOI 10.1016/j.atmosres.2011.06.013, 102, 99-109

[16] Sahai, A. K., R. Chattopadhyay and B. N. Goswami (2008), A SST based large multi-model ensemble forecasting system for Indian summer monsoon rainfall, Geophysical Research Letters, 35, L19705, doi:10.1029/2008GL035461

[17] S K Roy Bhowmik, V R Durai(2012), Development of multimodel ensemble based district level medium range rainfall, Indian Academy of Science, 121, no. 2 , 273285 .

[18] Suzuki,R., S. K. Behera, S. Iizuka, and T. Yamagata (2004), Indian Ocean subtropical dipole simulated using a coupled general circulation model, Geophysical Research Letters, 1091-18 doi:10.1029/2003JC001974
Harrison, M., Troccoli, A., Anderson, D.L.T. and Mason, S.,J.), NATO Science Series, Springer, Dordrecht, Netherlands, 82 205-234.

[12] Palmer, T.N. and others (2004), Development of a European multi-model ensemble system for seasonal to

[19] T. N. Krishnamurti, C. M. Kishtawal, Zhan Zhang, Timothy LaRow, David Bachiochi, and Eric Williford (2000), Multimodel Ensemble Forecasts for Weather and Seasonal Climate, journal of climate, American Meteorological Society, vol. 13 pg. 4196-4216

[20] Vladimir M. Krasnopolsky and Ying Lin (2012), “A Neural Network Nonlinear Multimodel Ensemble to Improve Precipitation Forecasts over Continental US," Advances in Meteorology, Article ID 649450, 11 pages, 2012. doi: $10.1155 / 2012 / 649450$

[21] W.T Yun, L.Stefanova, A.K Mitra, T.S.V. Vijaya Kumar, W. Dewar and T.N. Krishnamurti (2004), MultiModel Synthetic superensemble Algorithm for seasonal climate prediction using DEMETER Forecasts, Tellus, DEMETER

[22] W.W Hsieh. and Tang B. (1998). Applying neural network models to prediction and data analysis in meteorology and oceanography. Bulletin of American Meteorological Society, Vol.79 1855-1870.

[23] Y. Radhika and M. Shashi (2009), Atmospheric Temperature Prediction using Support Vector Machines, International Journal of Computer Theory and Engineering, Vol. 1, 1793-8201

[24] Zhang, G.P. and V.L. Berardi (2001). Time series forecasting with neural network ensembles: an application for exchange rate prediction. Operational Research Society, vol. 52, pg 652-664 